Georgian Mathematical Journal

Volume 14 (2007), Number 4, 699-710

\title{
POSITIVE SOLUTIONS FOR NEUTRAL DIFFERENCE EQUATIONS WITH CONTINUOUS ARGUMENTS
}

\author{
XIANYI LI
}

\begin{abstract}
Some "sharp" conditions are established for a kind of linear neutral difference equations with continuous arguments not to possess eventually positive solutions. The existence and asymptotic behavior are obtained for positive solutions of the kind of equations. The results for linear cases are further extended to nonlinear ones. A comparison principle, which is a necessary and sufficient condition, for linear equations not to possess eventually positive solutions is also presented.
\end{abstract}

2000 Mathematics Subject Classification: 39A10.

Key words and phrases: Neutral difference equation, existence, nonexistence, asymptotic behavior, comparison principle, positive solution, continuous argument.

\section{INTRODUCTION}

Investigations of oscillation and nonoscillation for neutral difference equations have been in rapid progress in the past few years. Various applications have been found, see, e.g., [1-3] and the references cited therein. As to the study of the qualitative properties of difference equations, most of the literatures deals with the case where variables are discrete, see the monographs $[1,7]$ and the papers [10-15]. There are only a few papers, where the case of continuous variables is considered $[4,5,6]$. Even less is known about the study of the oscillation and nonoscillation of linear, especially of nonlinear, neutral difference equations with continuous arguments.

In this paper we consider the following linear neutral difference equation with continuous arguments

$$
\nabla_{\tau}(y(t)-p(t) y(t-\tau))+q(t) y(t-\sigma \tau)=0,
$$

a more general linear neutral difference equation

$$
\nabla_{\tau}(y(t)-p(t) y(t-\tau))+\sum_{i=1}^{m} q_{i}(t) y\left(t-\sigma_{i} \tau\right)=0
$$

and the nonlinear neutral difference equation

$$
\nabla_{\tau}(y(t)-p(t) y(t-\tau))+q(t) \prod_{i=1}^{m}\left|y\left(t-\sigma_{i} \tau\right)\right|^{\alpha_{i}} \operatorname{sign} y\left(t-\sigma_{i} \tau\right)=0,
$$

where $\nabla_{\tau}$ is a backward difference operator defined by $\nabla_{\tau} y(t)=y(t)-y(t-\tau)$, $p(t) \in C\left(\left[t_{0}, \infty\right), R^{+}=[0, \infty)\right), q(t), q_{i}(t) \in C\left(\left[t_{0}, \infty\right),(0, \infty)\right), \sigma, \sigma_{i}, \tau \in$ 
$N=\{0,1,2, \ldots\}$ are constants and $\sigma, \sigma_{i}>\tau>0, i=1,2, \ldots, m, \alpha_{i} \geq 0$ and $\sum_{i=1}^{m} \alpha_{i}=1$.

On account of $y(t)$ being a solution of equation (1) (or $(2),(3)),-y(t)$ is also a solution of equation (1) (or (2), (3)). So, if there exists an eventually positive solution of equation (1) (or (2), (3)), then there also exists an eventually negative solution of equation (1) (or (2), (3)), i.e., there exist two nonoscillatory solutions of equation (1) (or (2), (3)), and if there does not exist an eventually positive solution of equation (1) (or (2), (3)), then there does not exist an eventually negative solution of equation (1) (or (2), (3)), either. That is to say then, all solutions of equation (1) (or (2), (3)) are oscillatory. Therefore, the study for oscillation and nonoscillation of equation (1) (or (2), (3)) is equivalent to that for the existence and nonexistence of eventually positive solutions of equation (1) (or $(2),(3))$.

When considering the oscillation of equation (1) (or (2) or (3)) with discrete arguments, namely, $t \in N, 0 \leq p(t) \leq 1$ is generally required in most known papers, e.g., [3]. In this paper, we get rid of the restriction and permit the interval for $p$ to take values in a very wide range.

To the best of our knowledge, for $p(t) \neq 0$ no results have so far been obtained for the oscillation and nonoscillation of equation (1), especially for equations (2) and (3), which is the main reason why we study in this paper the existence and nonexistence of positive solutions for the above three equations. Furthermore, our results are "sharp" in the sense that when the coefficients in the equations are constants, the sufficient conditions become the necessary and sufficient ones.

Equation (2) can be regarded as a discrete analogue of the neutral differential equation

$$
\frac{d}{d t}[y(t)-p(t) y(t-\tau)]+\sum_{i=1}^{m} q_{i}(t) y\left(t-\sigma_{i}\right)=0
$$

for which, see the papers by Y. Zhou [8], J. Sun and J. Wang [9].

For simplicity, put

$$
T=\max \left\{\sigma_{1}, \ldots, \sigma_{m}\right\} \tau \text { and } \quad \sigma^{*}=\min \left\{\sigma_{1}, \ldots, \sigma_{m}\right\} .
$$

Define the condition $(\mathrm{H})$ as follows.

$(\mathrm{H})$ Either the function $p(t)$ has arbitrarily large zeros or there exists a sufficiently large $s_{0} \geq t_{0}$ such that $p(s)>0$ for $s \geq s_{0}$ and, moreover, for any $k \in N$ and $s \geq s_{0}$

$$
\sum_{k=0}^{\infty}\left[\prod_{i=0}^{k} p(s+i \tau)\right]^{-1}=\infty
$$

By a solution $y(t)$ of equation (1), we mean a continuous function $y \in C\left(\left[t_{0}-\right.\right.$ $\sigma \tau, \infty), R$ ) which satisfies equation (1) for $t \geq t_{0}$. Solutions of equations (2) and (3) can be analogously defined.

As it is customary, a solution $y(t)$ of equation (1) (or (2), (3)) is said to be oscillatory if it is neither eventually positive nor eventually negative. Otherwise, 
a solution is called nonoscillatory. Equation (1) (or (2), (3)) is called oscillatory if every solution of it oscillates.

The arrangement of this paper is as follows. In Section 2 we state a useful lemma. In Section 3, we first deal in detail with the nonexistence of positive solutions of equation (1); then, we apply the idea to equations (2) and (3) to obtain a series of interesting results; finally, we consider the cases where the coefficients in Eqs (1) and (2) are constants, which manifests that our results are "sharp". The existence and asymptotic behavior of positive solutions of equation (2) is considered in Section 4. A comparison principle for the nonexistence of positive solutions of equation (2) is derived in Section 5, which is a necessary and sufficient condition.

\section{LEMMA}

Before stating our main results we need the following lemma which is very useful in proving our results.

Lemma 1. Suppose that the condition $(\mathrm{H})$ holds. Let $y(t)$ be an eventually positive solution of equation (1) (or (2), (3)). Set

$$
x(t)=y(t)-p(t) y(t-\tau) .
$$

Then eventually

$$
\nabla_{\tau} x(t)<0, \quad x(t)>0 .
$$

Proof. Assume that $y(t)$ is an eventually positive solution of equation (1). (The proof for the case where $y(t)$ is an eventually positive solution of equation (2) or equation (3) is similar and will be omitted.) Then there exists $t_{1} \geq t_{0}$ such that $y(t)>0, y(t-\tau)>0, y(t-\sigma \tau)>0$ for $t \geq t_{1}$. From (4) and equation (1) we know that

$$
\nabla_{\tau} x(t)=-q(t) y(t-\sigma \tau)<0 \quad \text { for } \quad t \geq t_{1} .
$$

Therefore if (5) does not hold eventually, then there exists a sufficiently large $t_{2} \geq \max \left\{s_{0}, t_{1}\right\}$ such that $x\left(t_{2}\right)<0$. Put $c=-x\left(t_{2}\right)$. Then it follows from (6) that

$$
-c=x\left(t_{2}\right)>x\left(t_{2}+\tau\right)>x\left(t_{2}+2 \tau\right)>x\left(t_{2}+3 \tau\right)>\cdots,
$$

i.e., $x\left(t_{2}+k \tau\right) \leq-c$ for any $k \in N$. If for some $k \in N, p\left(t_{2}+k \tau\right)=0$, then $y\left(t_{2}+k \tau\right)=x\left(t_{2}+k \tau\right) \leq-c<0$. A contradiction. So, for any $k \in N$, we have $p\left(t_{2}+k \tau\right)>0$. Accordingly,

$$
\begin{aligned}
y\left(t_{2}+k \tau\right)= & x\left(t_{2}+k \tau\right)+p\left(t_{2}+k \tau\right) y\left(t_{2}+(k-1) \tau\right) \\
\leq & p\left(t_{2}+k \tau\right) y\left(t_{2}+(k-1) \tau\right)-c \\
\leq & p\left(t_{2}+k \tau\right) p\left(t_{2}+(k-1) \tau\right) \cdots p\left(t_{2}+\tau\right) y\left(t_{2}\right)-c\left[1+p\left(t_{2}+k \tau\right)\right. \\
& +p\left(t_{2}+k \tau\right) p\left(t_{2}+(k-1) \tau\right)+\cdots \\
& \left.+p\left(t_{2}+k \tau\right) p\left(t_{2}+(k-1) \tau\right) \cdots p\left(t_{2}+2 \tau\right)\right]
\end{aligned}
$$




$$
=\left[\prod_{i=1}^{k} p\left(t_{2}+i \tau\right)\right]\left\{y\left(t_{2}\right)-c \sum_{i=1}^{k} \prod_{j=1}^{i}\left[p\left(t_{2}+j \tau\right)\right]^{-1}\right\}
$$

which, together with the hypothesis $(\mathrm{H})$, implies that $y\left(t_{2}+k \tau\right)<0$ for $k$ sufficiently large. This is also a contradiction. Hence, the proof of the proposition is complete.

\section{Nonexistence of Positive Solutions}

Our main results in this section are as follows.

Theorem 1. Assume that the condition $(\mathrm{H})$ holds. Furthermore,

$$
\inf _{t \geq t_{0}, \lambda>0}\left\{\frac{p(t-\sigma \tau) q(t)}{q(t-\tau)}(1+\lambda)+\frac{1}{\lambda} q(t)(1+\lambda)^{\sigma}\right\}>1 .
$$

Then there do not exist eventually positive solutions of equation (1).

Proof. Suppose the opposite that there exists an eventually positive solution $y(t)$ of equation (1). Then there exists a $t_{1} \geq t_{0}$ such that $y(t)>0, y(t-\tau)>0$, $y(t-\sigma \tau)>0$, for $t \geq t_{1}$. Let $x(t)$ be defined as (4). It is clear from Lemma 1 that there exists a sufficiently large $t_{2} \geq \max \left\{s_{0}, t_{1}\right\}$ such that (5) holds for $t \geq t_{2}$. Set

$$
u(t)=\frac{-\nabla_{\tau} x(t)}{x(t)}, \quad t \geq t_{2}
$$

Then

$$
u(t)>0, \quad \frac{x(t-\tau)}{x(t)}=1+u(t), \quad t \geq t_{2},
$$

and for any $n_{1}, n_{2} \in N$ with $n_{2}>n_{1}$ and $t \geq t_{2}+n_{2} \tau$,

$$
\frac{x\left(t-n_{2} \tau\right)}{x\left(t-n_{1} \tau\right)}=\prod_{k=n_{1}}^{n_{2}-1} \frac{x(t-(k+1) \tau)}{x(t-k \tau)}=\prod_{k=n_{1}}^{n_{2}-1}[1+u(t-k \tau)] .
$$

So, by equations (1), (4), (8), (9) and (10) we have, for $t \geq t_{3}=t_{2}+(\sigma+1) \tau$,

$$
\begin{aligned}
u(t) & =\frac{q(t)}{x(t)} y(t-\sigma \tau)=\frac{q(t)}{x(t)}[x(t-\sigma \tau)+p(t-\sigma \tau) y(t-\tau-\sigma \tau)] \\
& =q(t) \frac{x(t-\sigma \tau)}{x(t)}+\frac{q(t) p(t-\sigma \tau)}{q(t-\tau)} \frac{q(t-\tau) y(t-\tau-\sigma \tau)}{x(t-\tau)} \frac{x(t-\tau)}{x(t)} \\
& =q(t) \prod_{k=0}^{\sigma-1}[1+u(t-k \tau)]+\frac{q(t) p(t-\sigma \tau)}{q(t-\tau)} u(t-\tau)[1+u(t)] .
\end{aligned}
$$

We now show that $\liminf _{t \rightarrow \infty} u(t)>0$. Otherwise, there exists a sequence $\left\{\xi_{n}\right\} \subset N$ such that $\xi_{n} \rightarrow \infty, u\left(\xi_{n}\right) \rightarrow 0$ as $n \rightarrow \infty$ and $u\left(\xi_{n}\right)=\min \left\{u(t): t_{0} \leq t \leq \xi_{n}\right\}$. It follows from (11) that

$$
u\left(\xi_{n}\right) \geq q\left(\xi_{n}\right) \prod_{k=0}^{\sigma-1}\left[1+u\left(\xi_{n}\right)\right]+\frac{q\left(\xi_{n}\right) p\left(\xi_{n}-\sigma \tau\right)}{q\left(\xi_{n}-\tau\right)} u\left(\xi_{n}\right)\left[1+u\left(\xi_{n}\right)\right]
$$




$$
=\frac{p\left(\xi_{n}-\sigma \tau\right) q\left(\xi_{n}\right)}{q\left(\xi_{n}-\tau\right)} u\left(\xi_{n}\right)\left[1+u\left(\xi_{n}\right)\right]+q\left(\xi_{n}\right)\left[1+u\left(\xi_{n}\right)\right]^{\sigma}
$$

and so

$$
\frac{p\left(\xi_{n}-\sigma \tau\right) q\left(\xi_{n}\right)}{q\left(\xi_{n}-\tau\right)}\left[1+u\left(\xi_{n}\right)\right]+\frac{1}{u\left(\xi_{n}\right)} q\left(\xi_{n}\right)\left[1+u\left(\xi_{n}\right)\right]^{\sigma} \leq 1 .
$$

This contradicts (7). Therefore $a=\liminf _{t \rightarrow \infty} u(t)>0$. In view of condition (7) we find that there exists a positive number $\beta$ such that

$$
\inf _{t \geq t_{0}, \lambda>0}\left\{\frac{p(t-\sigma \tau) q(t)}{q(t-\tau)}(1+\lambda)+\frac{1}{\lambda} q(t)(1+\lambda)^{\sigma}\right\}>\beta>1 .
$$

Also, for a sufficiently large $t_{4}>t_{3}, u(t)>\frac{a}{\beta}$ for $t \geq t_{4}$. Then by (11) we see that

$$
u(t) \geq \frac{p(t-\sigma \tau) q(t)}{q(t-\tau)} \frac{a}{\beta}\left(1+\frac{a}{\beta}\right)+q(t)\left(1+\frac{a}{\beta}\right)^{\sigma}
$$

for $t \geq t_{5}=t_{4}+(\sigma-1) \tau$. One can further derive

$$
\inf _{t \geq t_{5}}\left\{\frac{p(t-\sigma \tau) q(t)}{q(t-\tau)}\left(1+\frac{a}{\beta}\right)+\frac{1}{\frac{a}{\beta}} q(t)\left(1+\frac{a}{\beta}\right)^{\sigma}\right\} \leq \beta,
$$

which is contrary to (12). Hence the proof is complete.

Theorem 1 readily implies

Corollary 1. If condition (7) in Theorem 1 is replaced by

$$
\liminf _{t \rightarrow \infty}\left\{\frac{p(t-\sigma \tau) q(t)}{q(t-\tau)}+q(t)\right\}>1,
$$

then there exist no eventually positive solutions of equation (1).

Next, we will apply the idea in Theorem 1 to equations (2) and (3).

Theorem 2. Suppose that the condition $(\mathrm{H})$ holds and there exists a function $h \in C\left(\left[t_{0}, \infty\right), R^{+}\right)$such that

$$
h(t) \leq \frac{p\left(t-\sigma_{i} \tau\right) q_{i}(t)}{q_{i}(t-\tau)}, \quad t \geq t_{0}, \quad i=1,2, \ldots, m
$$

and

$$
\inf _{t \geq t_{0}, \lambda>0}\left\{\frac{1}{\lambda} \sum_{i=1}^{m} q_{i}(t)(1+\lambda)^{\sigma_{i}}+h(t)(1+\lambda)\right\}>1
$$

Then there exist no eventually positive solutions of equation (2).

Proof. We only need to notice that (11) now takes the form

$$
\begin{aligned}
u(t) & =\frac{1}{x(t)} \sum_{i=1}^{m} q_{i}(t) y\left(t-\sigma_{i} \tau\right) \\
& =\frac{1}{x(t)} \sum_{i=1}^{m} q_{i}(t)\left[x\left(t-\sigma_{i} \tau\right)+p\left(t-\sigma_{i} \tau\right) y\left(t-\sigma_{i} \tau-\tau\right)\right]
\end{aligned}
$$




$$
\begin{aligned}
& =\frac{1}{x(t)}\left[\sum_{i=1}^{m} q_{i}(t) x\left(t-\sigma_{i} \tau\right)+\sum_{i=1}^{m} \frac{q_{i}(t) p\left(t-\sigma_{i} \tau\right)}{q_{i}(t-\tau)} q_{i}(t-\tau) y\left(t-\tau-\sigma_{i} \tau\right)\right] \\
& \geq \sum_{i=1}^{m} q_{i}(t) \frac{x\left(t-\sigma_{i} \tau\right)}{x(t)}+h(t) \frac{-\nabla_{\tau} x(t-\tau)}{x(t-\tau)} \frac{x(t-\tau)}{x(t)} \\
& =\sum_{i=1}^{m} q_{i}(t) \prod_{k=0}^{\sigma_{i}-1}[1+u(t-k \tau)]+h(t) u(t-\tau)[1+u(t)] .
\end{aligned}
$$

The rest of the proof is similar to that of Theorem 1 and therefore is omitted.

By Theorem 2 it is clear that the result below is true.

Corollary 2. Let condition (14) in Theorem 2 be replaced by

$$
\liminf _{t \rightarrow \infty}\left\{\sum_{i=1}^{m} q_{i}(t)+h(t)\right\}>1
$$

Then there exist no eventually positive solutions of equation (2).

Theorem 3. Suppose that the condition $(\mathrm{H})$ holds. Then

$$
\inf _{t \geq t_{0}, \lambda>0}\left\{\frac{1}{\lambda} q(t)\left[\prod_{i=1}^{m}\left((1+\lambda)^{\sigma_{i}}\right)^{\alpha_{i}}\right]+\frac{q(t)}{q(t-\tau)}\left[\prod_{i=1}^{m} p^{\alpha_{i}}\left(t-\sigma_{i} \tau\right)\right](1+\lambda)\right\}>1
$$

implies that there exist no eventually positive solutions of equation (3).

Proof. We only need to note that (11) now takes the form

$$
\begin{aligned}
u(t)= & \frac{q(t)}{x(t)} \prod_{i=1}^{m} y^{\alpha_{i}}\left(t-\sigma_{i} \tau\right)=\frac{q(t)}{x(t)} \prod_{i=1}^{m}\left[x\left(t-\sigma_{i} \tau\right)+p\left(t-\sigma_{i} \tau\right) y\left(t-\sigma_{i} \tau-\tau\right)\right]^{\alpha_{i}} \\
\geq & \frac{q(t)}{x(t)}\left[\prod_{i=1}^{m} x^{\alpha_{i}}\left(t-\sigma_{i} \tau\right)+\prod_{i=1}^{m} p^{\alpha_{i}}\left(t-\sigma_{i} \tau\right) \prod_{i=1}^{m} y^{\alpha_{i}}\left(t-\tau-\sigma_{i} \tau\right)\right] \\
= & q(t) \prod_{i=1}^{m}\left(\prod_{k=0}^{\sigma_{i}-1}[1+u(t-k \tau)]\right)^{\alpha_{i}} \\
& +\frac{q(t)}{q(t-\tau)}\left[\prod_{i=1}^{m} p^{\alpha_{i}}\left(t-\sigma_{i} \tau\right)\right] u(t-\tau)[1+u(t)] .
\end{aligned}
$$

The rest of the proof is similar to that of Theorem 1 and thus is omitted.

In view of Theorem 3, the following conclusion holds.

Corollary 3. Assume that condition (15) in Theorem 3 is replaced by

$$
\liminf _{t \rightarrow \infty}\left\{q(t)+\frac{q(t)}{q(t-\tau)}\left[\prod_{i=1}^{m} p^{\alpha_{i}}\left(t-\sigma_{i} \tau\right)\right]\right\}>1 .
$$

Then there exist no eventually positive solutions of equation (3). 
We are now in a position to investigate the oscillation of the special case of equation (1), i.e., the following linear neutral difference equation with constant coefficients

$$
\nabla_{\tau}(y(t)-p y(t-\tau))+q y(t-\sigma \tau)=0
$$

where $0 \leq p \leq 1$ and $q>0$. Obviously, $p$ satisfies the previous condition $(\mathrm{H})$. We have the following result.

Theorem 4. The necessary and sufficient condition for equation $\left(1^{*}\right)$ not to possess eventually positive solutions is

$$
p \lambda(1+\lambda)+q(1+\lambda)^{\sigma}-\lambda>0, \quad \lambda>0 .
$$

Proof. The sufficiency can be directly derived from Theorem 1. So, it suffices to prove the necessity. Assume that there exist no eventually positive solutions of equation $\left(1^{*}\right)$, whereas there is a $\lambda_{0} \in(0, \infty)$ such that

$$
p \lambda_{0}\left(1+\lambda_{0}\right)+q\left(1+\lambda_{0}\right)^{\sigma}-\lambda_{0} \leq 0
$$

Set

$$
F(\lambda)=p \lambda(1+\lambda)+q(1+\lambda)^{\sigma}-\lambda, \quad \lambda>0 .
$$

Then $F\left(\lambda_{0}\right) \leq 0$. Also, $\lim _{\lambda \rightarrow \infty} F(\lambda)=\infty$. Hence there exists $\lambda_{1}$ satisfying $\lambda_{0} \leq \lambda_{1}<\infty$ such that $F\left(\lambda_{1}\right)=0$. Set $y(t)=\left(1+\lambda_{1}\right)^{-t / \tau}, t \geq t_{0}$, which is an eventually positive solution of equation $\left(1^{*}\right)$. A contradiction.

Similarly, according to equation (2), we study the following neutral difference equation

$$
\nabla_{\tau}(y(t)-p y(t-\tau))+q \sum_{i=1}^{m} y\left(t-\sigma_{i} \tau\right)=0
$$

where the assumptions for $p, q$ are the same as in equation $\left(1^{*}\right)$. We obtain a conclusion similar to Theorem 4 that reads as follows.

Theorem 5. The necessary and sufficient condition for equation $\left(2^{*}\right)$ not to possess eventually positive solutions is

$$
p \lambda(1+\lambda)+q \sum_{i=1}^{m}(1+\lambda)^{\sigma_{i}}-\lambda>0, \quad \lambda>0 .
$$

Proof. The proof is similar to that of Theorem 4 and thus is omitted.

Remark 1. Theorems 4 and 5 imply that the conditions in Theorem 1 and Theorem 2 are the "sharp" ones.

\section{Existence of Positive Solutions}

In this section we will state some results for the existence and asymptotic behavior for positive solutions of equation (2). The main results are as follows. 
Theorem 6. Suppose that $\limsup _{t \rightarrow \infty} \sum_{i=1}^{m} q_{i}(t)<\infty$. If the difference inequality

$$
p(t) y(t-\tau)+\sum_{k=1}^{\infty} \sum_{i=1}^{m} q_{i}(t+k \tau) y\left(t-\sigma_{i} \tau+k \tau\right) \leq y(t), \quad t \geq t_{0}
$$

has a continuous positive solution $Y(t) \in C\left(\left[t_{0}-T+\tau, \infty\right),(0, \infty)\right)$ satisfying $\lim _{t \rightarrow \infty} Y(t)=0$, then equation (2) has a positive solution $x(t) \in C\left(\left[t_{0}-T+\right.\right.$ $\tau, \infty),(0, \infty))$ satisfying $0<x(t) \leq Y(t)$ for $t \geq t_{0}-T+\tau$.

Proof. Choose $T^{*}>t_{0}-T+\tau$ sufficiently large such that $Y\left(T^{*}\right)=$ $\min _{t_{0}-T+\tau \leq t \leq T^{*}} Y(t)$ and $Y(t)>Y\left(T^{*}\right)$ for $t \in\left[t_{0}-T+\tau, T^{*}\right)$. Define a function set by

$$
\Omega=\left\{\omega(t) \in C\left(\left[t_{0}-T+\tau, \infty\right),[0, \infty)\right): 0 \leq \omega(t) \leq Y(t), t \geq t_{0}-T+\tau\right\}
$$

and an operator on $\Omega$ as follows

$$
(S \omega)(t)=\left\{\begin{array}{l}
p(t) \omega(t-\tau)+\sum_{k=1}^{\infty} \sum_{i=1}^{m} q_{i}(t+k \tau) \omega\left(t-\sigma_{i} \tau+k \tau\right), \quad t \geq T^{*}, \\
(S \omega)\left(T^{*}\right)+Y(t)-Y\left(T^{*}\right), \quad t_{0}-T+\tau \leq t<T^{*} .
\end{array}\right.
$$

From (20) it is easy to see that $S \Omega \subset \Omega$. Clearly, $S$ is a monotonically nondecreasing operator. That is , for any given $\omega_{1}, \omega_{2} \in \Omega, \omega_{1}<\omega_{2}$ implies $S \omega_{1} \leq S \omega_{2}$. Now define a function sequence $\left\{x_{n}(t)\right\}$ on $\Omega$ as follows:

$$
x_{0}=Y, \quad x_{n}=S x_{n-1}, \quad n=1,2, \ldots .
$$

Then

$$
0 \leq x_{n}(t) \leq x_{n-1}(t) \leq \cdots \leq x_{0}(t) \leq Y(t), t \geq t_{0}-T+\tau .
$$

So, the limit $\lim _{n \rightarrow \infty} x_{n}(t)$ exists. Denote $\lim _{n \rightarrow \infty} x_{n}(t)=x(t)$. Then it is obvious that $0 \leq x(t) \stackrel{n \rightarrow \infty}{\leq} Y(t)$ for $t \geq t_{0}-T+\tau$. According to Lebesgue' Dominated Convergence Theorem, $x(t)$ satisfies the equation

$$
p(t) x(t-\tau)+\sum_{k=1}^{\infty} \sum_{i=1}^{m} q_{i}(t+k \tau) x\left(t-\sigma_{i}+k \tau\right)=x(t), \quad t \geq T^{*} .
$$

We now verify $x(t)$ to be a positive solution of equation (2). From $\lim _{t \rightarrow \infty} Y(t)=$ 0 and $0 \leq x(t) \leq Y(t)$ one can see that

$$
\lim _{t \rightarrow \infty} x(t)=0 .
$$

It follows from (21) that

$$
\begin{aligned}
\nabla_{\tau}(x(t)-p(t) x(t-\tau))=\nabla_{\tau}\left(\sum_{k=1}^{\infty} \sum_{i=1}^{m} q_{i}(t+k \tau) x\left(t-\sigma_{i} \tau+k \tau\right)\right) \\
=\sum_{k=1}^{\infty} \sum_{i=1}^{m} q_{i}(t+k \tau) x\left(t-\sigma_{i} \tau+k \tau\right) \\
\quad-\sum_{k=1}^{\infty} \sum_{i=1}^{m} q_{i}(t+(k-1) \tau) x\left(t-\sigma_{i} \tau+(k-1) \tau\right)
\end{aligned}
$$




$$
\begin{aligned}
= & \sum_{k=1}^{\infty}\left[\sum_{i=1}^{m} q_{i}(t+k \tau) x\left(t-\sigma_{i} \tau+k \tau\right)\right. \\
& \left.-\sum_{i=1}^{m} q_{i}(t+(k-1) \tau) x\left(t-\sigma_{i} \tau+(k-1) \tau\right)\right] .
\end{aligned}
$$

On the other hand, we have

$$
0 \leq \sum_{i=1}^{m} q_{i}(t+k \tau) x\left(t-\sigma_{i} \tau+k \tau\right) \leq \max _{1 \leq i \leq m} x\left(t-\sigma_{i} \tau+k \tau\right) \sum_{i=1}^{m} q_{i}(t+k \tau) \text {. }
$$

Then by the assumption $\limsup _{t \rightarrow \infty} \sum_{i=1}^{m} q_{i}(t)<\infty$ and $(22)$, it is easily seen from (24) that

$$
\lim _{k \rightarrow \infty} \sum_{i=1}^{m} q_{i}(t+k \tau) x\left(t-\sigma_{i} \tau+k \tau\right)=0
$$

which implies

$$
\begin{aligned}
\sum_{k=1}^{\infty}\left[\sum_{i=1}^{m} q_{i}(t+k \tau) x\left(t-\sigma_{i} \tau+k \tau\right)-\sum_{i=1}^{m} q_{i}(t\right. & \left.+(k-1) \tau) x\left(t-\sigma_{i} \tau+(k-1) \tau\right)\right] \\
& =-\sum_{i=1}^{m} q_{i}(t) x\left(t-\sigma_{i} \tau\right)
\end{aligned}
$$

As a consequence of (23) and (26), one can see that $x(t)$ is a solution of equation (2).

Next, it suffices to show that $x(t)>0$ as $t \geq t_{0}-T+\tau$. It is evident that $x(t)>0$ for $t_{0}-T+\tau \leq t<T^{*}$. Let $t^{*}=\inf \left\{t \geq T^{*}: x(t)=0\right\}$. Namely, $x(t)>0$ for $t \in\left[T^{*}, t^{*}\right)$ and $x\left(t^{*}\right)=0$. Whereas, by $(21)$,

$$
\begin{aligned}
x\left(t^{*}\right) & =p\left(t^{*}\right) x\left(t^{*}-\tau\right)+\sum_{k=1}^{\infty} \sum_{i=1}^{m} q_{i}\left(t^{*}+k \tau\right) x\left(t^{*}-\sigma_{i} \tau+k \tau\right) \\
& \geq \sum_{i=1}^{m} q_{i}\left(t^{*}+\tau\right) x\left(t^{*}-\sigma_{i} \tau+\tau\right)>0 .
\end{aligned}
$$

This is a contradiction and the proof is finished.

For nonoscillatory solutions of equation (1) we further have the following asymptotic result.

Theorem 7. Assume that the following two conditions hold:

(a) $p=\limsup _{t \rightarrow \infty} p(t)<1$,

(b) $\quad \sum_{k=1}^{\infty} \sum_{i=1}^{m} q_{i}(t+k \tau)=\infty$ for any fixed $t \geq t_{0}$.

Then any nonoscillatory solution $y(t)$ of equation (2) tends to 0 as $t \rightarrow \infty$. 
Proof. Let $y(t)$ be an eventually positive solution of equation (2). That is, there exists $t_{1} \geq t_{0}$ such that $y(t)>0, y\left(t-\sigma_{i} \tau\right)>0, i=1,2, \ldots, m, t \geq t_{1}$. Let $x(t)$ be defined by (4). Then (5) eventually holds. Indeed, from equation (2) we know that $\nabla_{\tau} x(t)=-\sum_{i=1}^{m} q_{i}(t) y\left(t-\sigma_{i} \tau\right)<0$ for $t \geq t_{1}$. Therefore If (5) does not hold eventually, then there exists $t_{2} \geq t_{1}$ such that $x\left(t_{2}\right)<0$. Denote $c=-x\left(t_{2}\right)$. Then it is easy to see that

$$
-c=x\left(t_{2}\right)>x\left(t_{2}+\tau\right)>x\left(t_{2}+2 \tau\right)>x\left(t_{2}+3 \tau\right)>\cdots,
$$

i.e., $x\left(t_{2}+k \tau\right) \leq-c$ for any $k \in N$.

By the definition of superior limit, there exists $k_{0} \in N$ such that for $t \geq$ $t_{2}+k_{0} \tau$ we have $p(t) \leq p+(1-p) / 2=(1+p) / 2<1$. Set $b_{k}=t_{2}+k_{0} \tau+k \tau$, $k \in N$ and $P=(1+p) / 2$. Then

$$
\begin{aligned}
y\left(b_{k}\right) & =x\left(b_{k}\right)+p\left(b_{k}\right) y\left(b_{k-1}\right) \leq p\left(b_{k}\right) y\left(b_{k-1}\right)-c \leq P y\left(b_{k-1}\right)-c \\
& \leq P^{k} y\left(t_{0}\right)-c\left(1+P+P^{2}+\cdots+P^{k-1}\right)=P^{k} y\left(t_{0}\right)-c \frac{1-P^{k}}{1-P},
\end{aligned}
$$

which implies that $y\left(b_{k}\right)<0$ for $k$ sufficiently large. This is a contradiction. Accordingly, for any fixed $t$, the limit $\lim _{k \rightarrow \infty} x(t+k \tau)$ exists. It follows that by equation (2) and (4)

$$
\nabla_{\tau} x(t)+\sum_{i=1}^{m} p_{i}(t) y\left(t-\sigma_{i} \tau\right)=0 .
$$

Replacing $t$ in (27) by $t+k \tau$ and then summing (27) from $k \geq t_{2}$ to $\infty$, one can easily see that the series $\sum_{k \geq t_{2}}^{\infty} \sum_{i=1}^{m} p_{i}(t+k \tau) y\left(t-\sigma_{i} \tau+k \tau\right)$ converges. Then, in view of (b), we obtain $\lim _{k \rightarrow \infty} \inf y(t+k \tau)=0$. Again, $0<x(t) \leq y(t)$. So $\lim _{t \rightarrow \infty} x(t)=0$.

To show $\lim _{t \rightarrow \infty} y(t)=0$, it suffices to prove $\limsup _{t \rightarrow \infty} y(t)=0$. Consider two possible cases.

Case (i). $y(t)$ is unbounded, i.e., $\limsup _{t \rightarrow \infty} y(t)=\infty$. Then there exists a sequence of points $\left\{t_{n}\right\}$ such that $t_{n} \geq t_{1}, n=1,2, \ldots, t_{n} \rightarrow \infty, y\left(t_{n}\right) \rightarrow \infty$ as $n \rightarrow \infty$ and $y\left(t_{n}\right)=\max _{t_{1} \leq t \leq t_{n}}\{y(t)\}, n=1,2, \ldots$ From (4) we have

$$
x\left(t_{n}\right)=y\left(t_{n}\right)-p\left(t_{n}\right) y\left(t_{n}-\tau\right) \geq\left[1-\limsup _{t \rightarrow \infty} p(t)\right] y\left(t_{n}\right) \rightarrow \infty,
$$

which is a contradiction.

Case (ii). $y(t)$ is bounded, i.e., $\lim \sup y(t)=d<\infty$. Thus there exists a sequence of points $\left\{u_{n}\right\}$ such that $u_{n} \geq t_{1}, n=1,2, \ldots, u_{n} \rightarrow \infty, y\left(u_{n}\right) \rightarrow d$ as $n \rightarrow \infty$ and $y\left(u_{n}\right)=\max _{t_{1} \leq t \leq u_{n}}\{y(t)\}, n=1,2, \ldots$ It suffices to show that $d=0$. If $d>0$, it is clear that (4) implies

$$
x\left(u_{n}\right)=y\left(u_{n}\right)-p\left(u_{n}\right) y\left(u_{n}-\tau\right) \geq\left[1-\limsup _{t \rightarrow \infty} p(t)\right] y\left(u_{n}\right)
$$


and so $0 \geq\left[1-\limsup _{t \rightarrow \infty} p(t)\right] d>0$, which is also a contradiction.

\section{Comparison Principle for Nonexistence of Positive Solution}

In this section we present a comparison principle, which is a necessary and sufficient condition for equation (2) not to possess positive solutions.

Theorem 8. Suppose that the conditions of Theorem 7 are true. Then the necessary and sufficient condition for equation (2) not to possess positive solutions is that the difference inequality

$$
\nabla_{\tau}(y(t)-p(t) y(t-\tau))+\sum_{i=1}^{m} q_{i}(t) y\left(t-\sigma_{i} \tau\right) \leq 0
$$

have no positive solutions.

Proof. The sufficiency is obvious. Now let us prove the necessity. Assume the opposite that inequality (28) has a positive solution $y(t) \in C\left(\left[t_{0}-T, \infty\right),(0, \infty)\right)$, and let $x(t)$ be defined as (4), i.e., $x(t)=y(t)-p(t) y(t-\tau)$, then, similarly to the proof of Theorem 7 , we have $\lim _{t \rightarrow \infty} y(t)=0, x(t)>0, \nabla_{\tau} x(t) \leq 0, \lim _{t \rightarrow \infty} x(t)=0$

and that the series $\sum^{\infty} \sum_{i=1}^{m} p_{i}(t+k \tau) y\left(t-\sigma_{i}+k \tau\right)$ converges. Noting equality (4), replacing $t$ in (28) by $t+k \tau$ and then summing (28) from $k=1$ to $\infty$, we obtain

$$
-x(t)+\sum_{k=1}^{\infty} \sum_{i=1}^{m} q_{i}(t+k \tau) y\left(t-\sigma_{i}+k \tau\right) \leq 0,
$$

or, equivalently,

$$
p(t) y(t-\tau)+\sum_{k=1}^{\infty} \sum_{i=1}^{m} q_{i}(t+k \tau) y\left(t-\sigma_{i}+k \tau\right) \leq y(t) .
$$

According to Theorem 6, that inequality (29) has a positive solution $y(t)$ satisfying $\lim _{t \rightarrow \infty} y(t)=0$ implies that equation (2) has a positive solution. A contradiction. We complete thereby the proof.

\section{ACKNOWLEDGEMENTS}

This work is supported by NNSF of China (10771094), the Foundation for "New Century '121' Talents in Hunan Province" and the Foundation for "Chief Professor of Mathematical Discipline in Hunan Province".

This work was done when the author was in the Department of Mathematics of University of Lille 1 in France as a post doctor supported by China Scholarship Council. The author is very thankful to Professor Changgui Zhang and Dr. Guoting Chen for their cordiality and hospitality. 


\section{REFERENCES}

1. R. P. Agarwal, Difference equations and inequalities. Theory, methods, and applications. Monographs and Textbooks in Pure and Applied Mathematics, 155. Marcel Dekker, Inc., New York, 1992.

2. B. G. Zhang, Positive solutions of a class of neutral equations. (Chinese) Acta Math. Appl. Sinica 19(1996), No. 2, 222-230.

3. X. H. TANG, J. S. Yu, and D. H. PEng, Oscillation and nonoscillation of neutral difference equations with positive and negative coefficients. Comput. Math. Appl. 39(2000), No. 7-8, 169-181.

4. Yuzhu ZhANG and JuRANG YAN, Oscillation criteria for difference equations with continuous arguments. (Chinese) Acta Math. Sinica 38(1995), No. 3, 406-411.

5. Yong ZHOU, Oscillation of difference equations with variable coefficients and continuous arguments. (Chinese) Economic Math. 13(1996), No. 1, 86-89.

6. J. H. Shen, Oscillation and comparison theorems of difference equations with continuous arguments and applications (Chinese) Chinese Sci. Bull. 41(16)(1996), No. 41, 14411444.

7. V. L. Kocic and G. LADAS, Global behavior of nonlinear difference equations of higher order with applications. Mathematics and its Applications, 256. Kluwer Academic Publishers Group, Dordrecht, 1993.

8. Y. ZHOU, Oscillation of neutral functional differential equations. Acta Math. Hungar. 86(2000), No. 3, 205-212.

9. J. Sun and J. Wang, On a conjecture of Chen and Huang. J. Math. Anal. Appl. 207(1997), No. 1, 61-72.

10. Xianyi Li and Deming Zhu, Global asymptotic stability in a rational equation. $J$. Difference Equ. Appl. 9(2003), No. 9, 833-839.

11. Xianyi Li and Deming Zhu, Global asymptotic stability for two recursive difference equations. Appl. Math. Comput. 150(2004), No. 2, 481-492.

12. Xianyi Li and Deming Zhu, Global asymptotic stability of a nonlinear recursive sequence. Appl. Math. Lett. 17(2004), No. 7, 833-838.

13. XiAnYi Li, Qualitative properties for a fourth-order rational difference equation. J. Math. Anal. Appl. 311(2005), No. 1, 103-111.

14. Xianyi Li and Deming Zhu, The rule of semicycle and global asymptotic stability for a fourth-order rational difference equation. Comput. Math. Appl. 49(2005), No. 5-6, 723730 .

15. Xianyi Li, Global behavior for a fourth-order rational difference equation. J. Math. Anal. Appl. 312(2005), No. 2, 555-563.

(Received 18.10.2005; revised 13.10.2007)

Author's addresses:

School of Mathematics and Physics

Nanhua University

Hengyang, Hunan 421001

P. R. China

Laboratoire Paul Painlevé

Université de Lille 1, Cité Scientifique

59655 Villeneuve d'Ascq, France

E-mail: xianyili6590@163.com 